\title{
Sudan IV Staining Method
}

National Cancer Institute

\section{Source}

National Cancer Institute. Sudan IV Staining Method. NCI Thesaurus. Code C78218.

The use of Sudan IV, a lysochrome (fat soluble dye) diazo dye, for the staining of lipids, triglycerides and lipoproteins on frozen paraffin sections. 